\section{PWE-118 DIAGNOSTIC AND THERAPEUTIC OUTCOMES IN GOBLET CELL APPENDICEAL NEUROENDCORINE TUMOURS}

doi:10.1136/gutjnl-2012-302514d.118

${ }^{1} \mathrm{M}$ Pericleous, ${ }^{*} \mathrm{~A}$ Baneke, ${ }^{1} \mathrm{C}$ Toumpanakis, ${ }^{2} \mathrm{~T}$ Meyer, ${ }^{1} \mathrm{M}$ E Caplin. ${ }^{1}$ Department of Gastroenterology, Neuroendocrine Tumours Unit, Royal Free Hospital, London, UK; ${ }^{2}$ Department of Oncology, Royal Free Hospital, London, UK

Introduction Goblet cell appendiceal neuroendocrine tumours (GCANETs), comprise approximately $6 \%$ of appendiceal neuroendocrine tumours (NETs) and share histologic features of both adenocarcinomas and NETs. We are presenting our data from 37 patients, focusing on diagnostic features, prognostic markers, treatment and survival.

Methods A retrospective analysis included 18 male and 19 female patients (mean age: 48.8; range: 19-73 years). Follow-up was complete (mean follow-up: 4.1 years).

Results Although majority of patients (69\%) presented with appendicitis, $15.5 \%$ had bowel obstruction and $15.5 \%$ atypical abdominal pain. $27 \%$ had metastases at presentation (one in lungs). Chromogranin-A, CEA and CA-125 were not significantly raised in these patients. Initial treatment was appendicectomy (26 patients) and $24 / 26$ had a subsequent prophylactic right hemicolectomy. Additional hysterectomy and bilateral oophorectomy was performed in six patients and four patients just underwent either single or bilateral oophorectomy. $12 \%$ had recurrence and all had Ki67 proliferation index $\geq 20 \%$. Octreoscan was negative, but FDGPET was positive in all these patients, and in patients with distal metastases at presentation. FOLFOX chemotherapy was given prophylactically in two patients with local lymph nodes resulting in no evidence of recurrence (Median $=8$ months) and in two patients with distal metastases resulting in only temporary disease stabilisation. 19\% have died with disease and again all had Ki67 $\geq 20 \%$.

Conclusion GCA-NETs may metastasise to the lungs (first report in literature). Ki67 $\geq 20 \%$ seems to be related with a worse prognosis. FDG-PET is the molecular imaging of choice. No optimal biomarkers or chemotherapy regimens are available to date.

Competing interests None declared.

\section{PWE-119 DUODENAL BULB BIOPSIES FOR DIAGNOSING ADULT COELIAC DISEASE: IS THERE AN OPTIMAL BIOPSY SITE?}

doi:10.1136/gutjnl-2012-302514d.119

${ }^{1} \mathrm{M}$ Kurien, ${ }^{*} \mathrm{~K}$ E Evans, ${ }^{1} \mathrm{~A} D$ Hopper, ${ }^{1} \mathrm{M} F$ Hale, ${ }^{2} S \mathrm{~S}$ Cross, ${ }^{1} \mathrm{D} S \mathrm{~S}$ Sanders. ${ }^{1}$ Department of Gastroenterology, Royal Hallamshire Hospital, Sheffield, UK; ${ }^{2}$ Department of Pathology, Royal Hallamshire Hospital, Sheffield, UK

Introduction There has been increasing interest in the role that duodenal bulb biopsies may have in helping to establish the diagnosis of coeliac disease. This study aims to determine whether a targeted duodenal bulb biopsy in addition to distal duodenal biopsies is the optimal strategy when trying to identify villous atrophy, comparing histological findings from different quadrants of the duodenal bulb.

Methods Patients undergoing oesophogastroduodenoscopy (OGD) were prospectively recruited from a single tertiary referral hospital in the UK between July 2010 and October 2011. Indications for biopsy included positive coeliac serology, family history of coeliac disease, chronic diarrhoea, iron deficiency anaemia, abdominal pain and weight loss. All patients recruited to the study had immunoglobulin A (IgA) endomysial antibody (EMA) and tissue transglutaminase (tTG) antibody measurements prior to undergoing their EGD. At endoscopy, eight duodenal biopsies were taken: four from the second part of the duodenum and four quadrantically from the bulb (3,6,9 and 12 o'clock). Each biopsy was graded according to the modified Marsh Criteria, with the optimal biopsy site in the bulb being evaluated by the ability to detect the presence and severity of villous atrophy.

Results A total of 77 patients were recruited (27 male (35\%), 50 female (65\%), median age 45, range 19-79) between July 2010 and November 2011. Of these, 28 (36\%) were found to have newly diagnosed coeliac disease and 49 were controls (64\%). Bulbar villous atrophy was identified in $96 \%$ of the coeliac patients, with five patients having villous atrophy confined to the bulb alone (Abstract PWE-119 table 1). The most severe degree of villous atrophy was detected when distal duodenal biopsies were taken in addition to a duodenal bulb biopsy from either the 9 or 12 o'clock position (sensitivity $96.4 \%, 95 \%$ CI $79.7 \%$ to $100 \%$ ). The difference between the 12 o'clock biopsy and the 3 o'clock biopsy in detecting the most severe villous atrophy was $92 \%(24 / 26)$ vs $65 \%(17 / 26)(p=0.04)$.

Abstract PWE-119 Table 1 Histology results

\begin{tabular}{lllllll}
\hline & $\mathbf{n}$ & $\begin{array}{l}\text { Coeliac } \\
\text { serology +ve }\end{array}$ & VA in D1 & VA in D2 & $\begin{array}{l}\text { VA in } \\
\text { D1 only }\end{array}$ & $\begin{array}{l}\text { VA in } \\
\text { D2 only }\end{array}$ \\
\hline Coeliac disease & 28 & $25(89 \%)$ & $26(93 \%)$ & $23(82 \%)$ & $5(18 \%)$ & $2(7 \%)$ \\
Control group & 49 & $12(24 \%)$ & 0 & 0 & 0 & 0
\end{tabular}

D1, Duodenal Bulb; D2, Second part of the duodenum; VA, Villous Atrophy.

Conclusion This study demonstrates the patchy appearance of villous atrophy that occurs within the duodenum. A targeted duodenal bulb biopsy from either the 9 or 12 o'clock position in addition to distal duodenal biopsies, may improve diagnostic yields by detecting the most severe villous atrophy within the duodenum.

Competing interests None declared.

\section{PWE-120 HOW DOES A BLOOD TRANSGLUTAMINASE-BASED RAPID TEST COMPARE TO CONVENTIONAL SEROLOGICAL MARKERS TO DETECT COELIAC DISEASE?}

doi:10.1136/gutjnl-2012-302514d.120

${ }^{1} \mathrm{M}$ Kurien, ${ }^{*}{ }^{1} \mathrm{~K}$ E Evans, ${ }^{1} \mathrm{I}$ Chalkiadakis, ${ }^{1} \mathrm{M}$ F Hale, ${ }^{1} \mathrm{~V}$ Courtice, ${ }^{1} \mathrm{~A} \mathrm{~J}$ Irvine, ${ }^{2} \mathrm{M}$ Hadjivassiliou, 'D S Sanders. ${ }^{1}$ Department of Gastroenterology, Royal Hallamshire Hospital, Sheffield, UK; ${ }^{2}$ Department of Neurology, Royal Hallamshire Hospital, Sheffield, UK

Introduction Coeliac disease may be missed at endoscopy. For this reason many centres take routine duodenal biopsies or have a low threshold for duodenal biopsy. While duodenal biopsies demonstrating villous atrophy remains the current gold standard, serological markers are widely used either alone or in combination (tissue transglutaminase (TTG)/endomysial antibody (EMA)) to help identify at risk individuals. However, these results may not be available at the time of endoscopy. Recently a whole blood transglutaminase-based rapid test has become available. This Point of Care Test (POCT) can be read in 5-10 min prior to endoscopy and could help in determining which patients having an endoscopy should have a duodenal biopsy. This strategy could also have costsaving implications if we could reduce the number of duodenal biopsies performed. This is the first study to assess the clinical utility of this POCT within the setting of endoscopy. Comparisons are made against current serological markers and duodenal biopsy. Methods Patients undergoing oesophogastroduodenoscopy (OGD) with duodenal biopsies were prospectively recruited between August 2010 and November 2011. Unselected patients undergoing endoscopy were concurrently serologically tested for IgA TTG, EMA, a total IgA immunoglobulin level and the transglutaminasebased rapid test. Sensitivity, specificity, positive predictive value (PPV) and negative predictive value (NPV) for the transglutaminasebased rapid test were calculated and comparisons made with TTG 